\title{
The Application of the Exhaustive Polling Theory in Intelligent Traffic System
}

\author{
Meng Yao Wang ${ }^{1, a}$, Hong Wei Ding ${ }^{1, b}$, Yi Fan Zhao ${ }^{1}$, Zhu Guan Liang ${ }^{1}$, Long Zheng Zhu ${ }^{1}$ \\ ${ }^{1}$ School of Informatics, Yunnan University, China \\ ayakazo@163.com, b dhw1964@163.com
}

\begin{abstract}
This paper presents a new use of exhaustive service polling system in the intelligent traffic light control system.Vehicles arrival rate is measured in the system. Through the relationship between arrival rate and mean waiting time, mean queue length in exhaustive service polling system, achieved an technology that intelligent adjust the length of traffic light time according to the arrive rate.The more arrive rate the longer green light time. With the intelligent control, the road capacity is more.

Keywords: polling system, exhaustive service, mean waiting time, mean queue length, intelligent traffic
\end{abstract}

\section{Introduction}

Intelligent transportation come into sight as one of the main solution to modern traffic pressure, gradually.Since 1868 the first traffic lights $^{[1]}$, traffic lights development has the changes of the red and blue traffic lights, red and green traffic lights, red, yellow and green traffic lights, and now, the intelligent traffic lights. The main rule of red yellow and green traffic light which is widely used now is the vehicles can go straight and turn left when the light is green except there is a mandatory forbidding sign in front of the intersection. And the vehicles that turn left or right must let the straight road vehicles and pedestrians go first. The vehicles which in the motorway is not allowed to over the sidewalk when the light is red. Yellow light mean to wait,vehicles must slow down when the light is yellow, but the vehicle which is very close to stop line and can't park safely can enter the intersection. And the main function is through the different colors of light indicates the specification that vehicles and pedestrians through the intersection, to ensure the safe passage of vehicles and pedestrians.
Intelligent traffic light $^{[2]}$ theory is not only focus on ensure the orderly safe passage of vehicles and pedestrians with the same rules of ordinary traffic lights, more is control the waiting time intelligently in order to improve the traffic efficiency under the background of increasing traffic pressure,

According to this principle, the main task of this paper is to design a intelligent traffic lights system which use the exhaustive service polling model. This system can adjust the mean time delay according to the arrive rate .To alleviate traffic pressure, and can save transportation resources.Through reasonable design the system function, the conversion of the red, yellow, green light have an accurate time interval which requires an automated and secure system to control .

\section{Specific design}

we design a traffic lights system which the timekeeping time during light can be adjusted intellectually according to the change of the traffic volume.Initial idea is to install photoelectric counter in all directions at the crossroads to count the vehicles, and then enter data into the computer central control system 
to analyze the data.The intersection traffic lights to ensure the safe passage of pedestrians and vehicles.There are red, green and yellow lights set up in each side .Red light means no thoroughfare, and green light means vehicles can thorough the intersection. There is yellow light for a few seconds when green to red, so that the vehicles could stop .

In this system,the exhaustive service ${ }^{[3]}$ be used in the two directions of the intersection.Means that the length of green light time in any road should conforms with the result which is calculated by the exhaustive service polling theory.On other word ,the length of red light time depend on anther direction's length of green light time. And there is one would never change over the traffic volume that is length of yellow light time.

\section{The exhaustive service polling model of traffic lights intelligent control system}

This traffic lights intelligent control system $^{[8]}$ is seem as a double queues single servers polling system. For practical purposes ,suppose, the direction of North and South is seen as a queuel with the arrival rate of vehicles which is $\lambda_{1}$, so as the direction of East and West.The $\beta$ means the service rate ${ }^{[9]}$ of the traffic light system. In this intelligent system $\beta$ is 0.5 ,mean there are one vehicle though the intersection during the $2 \mathrm{~s}$. The conversion rate[10] of traffic light system is the length of yellow light time in this system,and there $\gamma=1$ means that there are $4 \mathrm{~s}$ yellow light. The mean time delay and the mean queue length of each queues is figured out by the exhaustive service polling model which is $\mathrm{N}=2$.

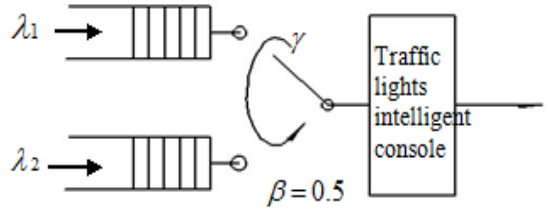

$\beta$ : The service rate of the traffice light system $\gamma$ : The conversion rate of the traffice light system $\lambda_{1}$ : The arrival rate of queuel $\lambda_{2}$ : The arrival rate of queue 2

Figure 1. The exhaustive service polling model of intelligent traffic lights control system

Apply this model to intelligent traffic lights control system, the mean time delay ${ }^{[11]}$ of queue 1 is the time of each vehicles waiting in
North and South, can be calculated by the exhaustive service polling system under $\mathrm{N}=2$.In order to keep the roads clear ,the road which has the more mean time delay need the longer green light time. So,the mean time delay in north and south, we calculate, equal the time of green light in north and south. So as West and East .

Whenever the mean time delay were calculated greater than 100 seconds, we set the green light time as 100 seconds. Whenever the mean time delay less than 100 seconds but longer than 10 seconds, the traffic lights would be controlled with the calculation results. The minimal green light time is 10 seconds.

\section{Queuing model and operation mode}

Assume that there are 2 roads in this intelligent traffic lights control system that using the exhaustive service polling system. And those 2 roads be serviced by the one traffic lights under the rules of exhaustive service polling system .System's logic loop service queuing model is shown in figure 1.Because of the queuing system is discussed in this paper is under the discrete time state , so the time line divided according to the time slot $\tau=1$ as the unit (concise analysis into consideration, take $\tau$ as unit time)

\section{1. parametric hypothesis}

(1)Each queues has the vehicles arrived into their queue space with independent and identically probability distribution at any time slot the distribution of probability generating function, the mean and variance are $A(Z)$ 、

$\lambda=A^{\prime}(1)$ and $\delta_{\lambda}^{2}=A^{\prime \prime}(1)+\lambda-\lambda^{2}$ respectively.

(2)The random variable of query conversion time between the two queues is subject to a probability distribution which is mutually independent and identically distributed,the distribution of probability generating function, the mean and variance are $R(Z) 、 \quad \gamma=R^{\prime}(1)$ and $\delta_{\gamma}^{2}=R^{\prime \prime}(1)+\gamma-\gamma^{2}$ respectively.

(3)The random variable of the time that each vehicles pass the crossroads is subject to a probability distribution which is mutually independent and identically distributed, the distribution of probability generating function, 
the mean and variance are $B(Z), \beta=B^{\prime}(1)$ and $\delta_{\beta}^{2}=B^{\prime \prime}(1)+\beta-\beta^{2}$ respectively.

(4)The queue space is big enough .The number of vehicles would not more than the queue space.

(5)For the vehicles that in the each queue, according to the principle of first-in-first-out.

\section{2.calculate of $\Pi_{1}\left(X_{1}, X_{2}, \ldots X_{i}, \ldots X_{i}\right)$}

Assuming that $i$ road $(i=1,2)$ accept service of intelligent traffic lights at $t_{n}$ (It' $\mathrm{s}$ multiple of $\tau$ ), the intelligent traffic lights would turn to service $i+1$ road after $i$ road send all of its information packets by the way of exhaustive service $i+1$ road is serviced at $t_{n+1}$ (It's s multiple of $\tau$ ).

Random variable $\xi_{i}(n)$ is defined as the number of vehicles in ${ }^{i}$ road at $t_{n}$, so the state of the queuing system at $t_{n}$ can be represented as $\xi_{1}(n), \xi_{2}(n), . \xi_{i}(n), ., \xi_{N}(n)$.

Here: ,

The probability generating function of $\Pi_{i}\left(x_{1}, x_{2}\right)$.

$$
\begin{aligned}
& G_{i}\left(z_{1}, z_{2}\right)=\sum_{X_{1}=0}^{\infty} \sum_{X_{2}=0}^{\infty} \prod_{i}\left(x_{1}, x_{2}\right) \bullet z_{1}^{x_{1}} z_{2}^{x_{2}} \\
& i=1, \quad 2 ; \quad \text { (1) }
\end{aligned}
$$

\section{3. calculate of $g_{i, S}(i, i)$}

If the road $i$ turn the green light and the vehicles in the road $i$ begin to pass the intersection at the $t_{n}$, and the all vehicles pass at the $t_{n_{1}}$. Because the exhaustive service ,there are $\xi_{i}\left(n_{1}\right)$ number of vehicles arrive in the road ${ }^{i}$ at the $t_{n_{1}}$,all of those vehicles pass the intersection at $t_{n_{2}}\left(t_{n} \leq t_{n_{1}}\right)$ . This process show as fig2.

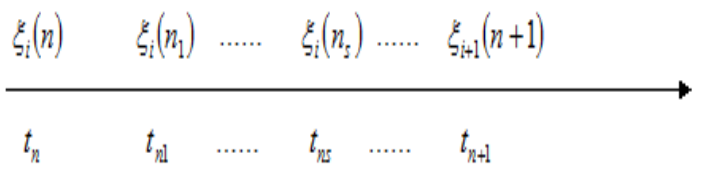

Figure 2. The process that pass all vehicles in road $i$

The generating function of system state equation at $t_{n}, t_{n_{1}}$ can be defined as before :

$$
\begin{aligned}
& g_{i, n_{s}}(i, i)=\rho^{2 s} g_{i}(i, i)+\frac{\rho^{s-1}\left(1-\rho^{s}\right)\left(\beta A^{\prime \prime}(1)+\lambda^{2} B^{\prime \prime}(1)\right)}{1-\rho} g_{i}(i) \\
& i=1,2 \quad s=1,2
\end{aligned}
$$

\section{4. calculate of $\bar{W}_{E}$}

We can get the mean waiting time:

$$
\bar{W}_{E}=\bar{W}_{i, 1}+\bar{W}_{i, 2}=\frac{1}{2}\left\{\frac{R^{\prime \prime}(1)}{\gamma}+\frac{1}{1-2 \rho}\left[\gamma+\rho+2 \lambda B^{\prime \prime}(1)\right]+\frac{\rho A^{\prime}(1)}{\chi^{2}(1-2 \rho)}\right\}
$$

\section{The simulation}

The simulation calculation and theoretical calculation take the same parameters. And in those simulation diagrams, we set the vertical axis narrows 10 times.

the horizontal axis load is $\rho=\beta \lambda$, and $\rho$ is proportional to the arrival rate $\lambda$.In this paper, we set the service rate $\beta=0.5$,means there are one vehicle though the intersection during the $2 \mathrm{~s}$.

In theory, whenever $\rho=1$ means this system would be on the brink of collapse.But in this paper, we set the maximum waiting time is $100 \mathrm{~s}$, so the $\rho$ have to less than 0.4 .On other words ,the $\lambda$ have to less than 0.8 means there are 8 vehicles though the intersection during the $10 \mathrm{~s}$.

The Fig3 and Fig4 reflect the theoretical value and the simulation values are the same.In those two diagrams we can find that the mean queue length and the mean time delay are proportional to load.Compare exhaustive service and gate service we can find that the mean queue length and the mean time delay of exhaustive service is shorter than the mean queue length and the mean time delay of gate service with the same system load or the same arrive rate (Because $\rho$ is proportional to the $\lambda)$.The shorter mean queue length and the shorter mean time delay mean that the shorter green light time be needed with the same 
vehicles arrive rate.So the intelligent traffic lights control system which use the exhaustive service polling system is better than the system which use gate service.

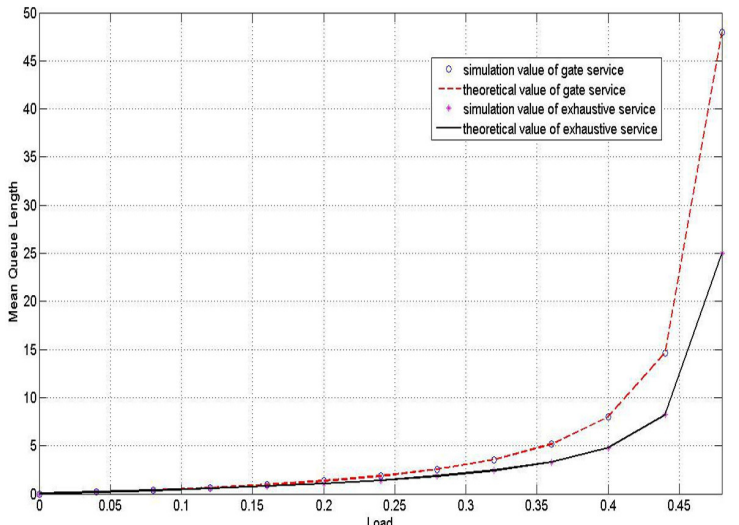

Figure 3. The simulation diagram of mean time delay

\section{Conclusions}

Different road has different arrival rate .In order to improve the road capacity, a intelligent control system is necessary in today.Though the exhaustive service polling system,we find the relationship that the the longer mean time delay because of the higher arrive rate.In the intelligent traffic light control system , the longer mean time delay means should set longer green light time to keep the road open.Different time has different arrive rate, in order to improve the road capacity, the time of traffic light has to adjust intellectually according to the arrive rate. The intelligent traffic light control system which use the exhaustive service polling system can keep the shorter waiting time with the same arrive rate.So this system can relieve the traffic congestion,improve the capacity of intersectio ns.

\section{References}

[1] Ding Hongwei, Zhao Dongfeng, Zhao Yifan. Packet delay analysis of continuoustime polling system with M-gated services[A]. Chengdu, P.R.China, August, 2010, Page(s):408-411.

[2] Qianlin Liu, Dongfeng Zhao, Dongming Zhou. An analytic model for enhancing IEEE 802.11 coordination function media access control protocol[J]. European Transactions on Telecommunications, 2011, 22(6):332-338.

[3] Jan-Pieter Dorsman, S. Borst, O. Boxma, Maria Vlasiou,Markovian polling systems with an application to wireless random-access networks[J].IEEE Trans. Eurandom Prepront Series, 2014, 5(6):1389-2355.

[4] Zhao Dongfeng, Ding Hongwei, Zhao Yifan, Wang Mingui. An Analytical Model of a Discrete-Time Polling MAC Protocol for Wireless LANs Using M-Gated Services[J]. Acta. Electronica Sinica, 2010, 38(7): 1495 1499. (in Chinese)

[5] Giambene, G.,Hadzic-Puzovic, S. Nonsaturated Performance Analysis for WiMAX Broadcast Polling Access[J]. IEEE Transactions on Vehicular Technology, 2013, 62(1):306-325.

[6] Qianlin Liu, Dongfeng Zhao, Dongming Zhou. An analytic model for enhancing IEEE 802.11 coordination function media access control protocol[J]. European Transactions on Telecommunications, 2011, 22(6):332-338.

[7] Liu Qianlin, Zhao Dongfeng, Zhao Yifan. An efficient priority service model with twolevel-polling scheme[J]. High Technology Letters, 2011, 17(3): 245-251.

[8] Khoshnevis, B., Wei Yu, Lostanlen, Y. Two-Stage Channel Quantization for Scheduling and Beamforming in Network MIMO Systems: Feedback Design and Scaling Laws[J]. IEEE Journal on Selected Areas in Communications, 2013, 31(10): 2028-2042.

[8] Yang Zhijun;Ding Hongwei;Chen Chuanglong.Exhaustive service and gated service polling system with two levels of $\mathrm{E}(\mathrm{x})$ characteristics analysis[J]; Acta Electronica Sinica ,2014,04:774-778.

[9] A.S. Salama, B.K. Saleh, M.M. Eassa, Intelligent cross road traffic management system (ICRTMS), in: 2nd International Conference on Computer Technology and Development, ICCTD, Cairo, Egypt, November 2010: 27 - 31 .

[10]Dimitrakopoulos, G. (2011) Intelligent transportation systems based on internetconnected vehicles: Fundamental research areas and challenges. 11th International Conference on ITS Telecommunications (ITST), Saint Peterburg, 23-25 August 2011, 145-1 